\title{
Anti-CD133-PE38-KDEL Fusion Protein
}

National Cancer Institute

\section{Source}

National Cancer Institute. Anti-CD133-PE38-KDEL Fusion Protein. NCI Thesaurus. Code C131828.

A fusion protein consisting of an anti-single-chain variable fragment (scFv) peptide sequence targeting the extracellular domain of human CD133 (prominin-1) (antiCD133scFV) and a deimmunized truncated form of Pseudomonas exotoxin A (38-kDa derivative of PE; PE38) where the five C-terminal amino acid residues have been replaced with the endoplasmic reticulum (ER) retention signal, KDEL, with potential antineoplastic activity. Upon administration of the anti-CD133-PE38-KDEL fusion protein, the antiCD133 scFV moiety targets and binds to CD133, which is expressed on a variety of tumor cells. Upon internalization of the receptor-fusion protein complex, the KDEL sequence targets the fusion protein to the ER, where the PE38 exotoxin portion then inhibits protein synthesis, which results in a reduction of proliferation of CD133-expressing tumor cells. CD133, a glycoprotein expressed by a variety of cancers and especially by cancer stem cells (CSCS), plays a key role in tumor initiation, proliferation and progression. 